Mens

Revue d'histoire intellectuelle et culturelle

mens

Germain Lacasse, Johanne Massé et Bethsabée Poirier. Le

diable en ville: Alexandre Silvio et l'émergence de la

modernité populaire au Québec, Montréal, Les Presses de

l’Université de Montréal, 2012, 299 p.

\title{
Harold Bérubé
}

Volume 13, numéro 2, printemps 2013

URI : https://id.erudit.org/iderudit/1025985ar

DOI : https://doi.org/10.7202/1025985ar

Aller au sommaire du numéro

Éditeur(s)

Centre de recherche en civilisation canadienne-française

ISSN

1492-8647 (imprimé)

1927-9299 (numérique)

Découvrir la revue

Citer ce compte rendu

Bérubé, H. (2013). Compte rendu de [Germain Lacasse, Johanne Massé et Bethsabée Poirier. Le diable en ville : Alexandre Silvio et l'émergence de la modernité populaire au Québec, Montréal, Les Presses de l'Université de Montréal, 2012, 299 p.] Mens, 13(2), 105-108. https://doi.org/10.7202/1025985ar 
les archives personnelles de Furet, il traite peu de sa vie privée, sauf pour rappeler ce que l'historien avait lui-même confié lors d'entrevues. On apprend que Furet perdit ses parents très jeune, qu'atteint de tuberculose il fut alité de 1950 à 1954, qu'il épousa trois femmes avec qui il eut deux enfants. "Je préfere la passion amoureuse à la passion politique ", admit-il lors d'une entrevue radiophonique. Historien des idées plutôt que sociologue des connaissances, Prochasson ne s'intéresse pas aux divers réseaux entretenus par Furet, aux rivalités académiques ou aux luttes de pouvoir internes à l'université. L'aurait-il fait qu'on aurait peut-être mieux compris pourquoi ses idées en vinrent à rayonner à ce point, surtout à l'extérieur de l'université. Cela dit, il montre bien le souci de Furet pour le grand public, son talent dans les médias, sa facilité à communiquer au plus grand nombre. Plus passeur que vulgarisateur, Furet croyait qu'il était possible " d'énoncer clairement et élégamment les résultats de la recherche historique, sans renoncer à une rigueur fondée plus objectivement. L'histoire a son vocabulaire, elle n'a pas besoin de son jargon" (cité p. 485). Seule lacune notable dans cet ouvrage instructif, on n'y trouve aucune bibliographie complète des ouvrages et articles de François Furet ni des études portant sur son œuvre.

- Éric Bédard

TÉLUQ

Germain Lacasse, Johanne Massé et Bethsabée Poirier. Le diable en ville : Alexandre Silvio et l'émergence de la modernité populaire au Québec, Montréal, Les Presses de l'Université de Montréal, 2012, 299 p.

Cet ouvrage d'histoire culturelle permet de découvrir la figure méconnue du bonimenteur, cette personne qui avait pour tâche, aux premières années du cinéma, de lire, de traduire ou de bonifier les intertitres qui facilitaient la compréhension des films muets. Comme l'expliquent les auteurs, c'est une figure qui a généralement eu une présence plus importante et durable dans les communautés 
minoritaires comme le Canada français. Plus spécifiquement, l'ouvrage s'intéresse à la trajectoire d'Alexandre Silvio, bonimenteur qui est, par la suite, devenu auteur, producteur et directeur de différents théâtres et qui a joué un rôle clé dans le développement du divertissement populaire dans le Montréal francophone des premières décennies du $\mathrm{Xx}^{\mathrm{e}}$ siècle.

À partir de sources malheureusement très fragmentaires et à la suite d'un travail de recherche et d'analyse visiblement minutieux, l'historien du cinéma Germain Lacasse et deux de ses étudiantes à la maîtrise, Johanne Massé et Bethsabée Poirier, parviennent à éclairer ce pan peu connu de la culture urbaine montréalaise. Le premier chapitre de l'ouvrage leur permet de mettre en contexte les activités de Silvio, et c'est probablement sur ce plan que se situe la principale faiblesse de l'ouvrage. Dans ce chapitre et dans ceux qui suivent, Lacasse et ses collaboratrices exagèrent nettement l'influence culturelle de l'Église catholique dans la métropole durant le premier tiers du $\mathrm{Xx}^{\mathrm{e}}$ siècle. Décrivant fréquemment leur bonimenteur et ses activités comme une sorte de bastion de liberté inattendu dans une cité écrasée par le joug d'une religion conservatrice et rigoriste, ils sous-estiment largement l'espace de liberté dont disposaient les Canadiens français catholiques de la métropole durant ces années. Ignorant l'évolution de l'historiographie québécoise des dernières décennies, ils opposent ainsi une "société évoluant selon les idéaux traditionalistes solidement entretenus par une élite cléricale [aux] créations audacieuses d'artistes qui exploitaient sur scène en jargon populaire les sujets les plus contemporains liés à la modernité " (p. 11). Le simple ajout, dans leur bibliographie, de l'ouvrage de Lucia Ferretti sur la paroisse SaintPierre Apôtre leur aurait permis de mesurer à quel point la capacité de l'Église à encadrer efficacement ses fidèles dans ce milieu était allée en déclinant depuis la deuxième moitié du XIX ${ }^{\mathrm{e}}$ siècle. La lecture de l'ouvrage d'E.-Martin Meunier et Jean-Philippe Warren sur le personnalisme, ou alors celui de Michael Gauvreau sur les origines catholiques de la Révolution tranquille, leur aurait également permis de constater qu'ils ne sont pas les premiers à " trouver" les racines 
de la Révolution tranquille dans les décennies qui précèdent l'élection du gouvernement de Jean Lesage. En d'autres mots, leur contribution n'est pas tant, comme ils se l'imaginent, d'avoir découvert une modernité populaire insoupçonnée dans le Montréal du premier tiers du $\mathrm{Xx}^{\mathrm{e}}$ siècle, que d'avoir mis au jour certaines des formes, très peu connues, qu'elle prenait dans les théâtres francophones de la métropole.

Les auteurs ont plus de succès lorsque vient le moment de situer la place, ingrate, qu'occupent les formes populaires de divertissement, tant chez la critique de l'époque que dans les recherches qui ont suivi et qui se sont longtemps concentrées sur les formes plus "nobles " de la culture canadienne-française. Mais, comme ils le montrent éloquemment dans les trois autres chapitres de l'ouvrage (autant d'actes subdivisés en différents tableaux), les formes culturelles étudiées ici représentent autant de façons, pour les classes populaires montréalaises, d'apprivoiser et de s'approprier la modernité urbaine.

Ainsi, le premier acte de leur démonstration leur permet d'explorer les débuts de différentes formes de divertissement populaire urbain. Il y a évidemment le cinéma, la censure qu'il suscite, les bonimenteurs qui, au contraire, accompagnent le spectateur dans son écoute, et le star-system qui s'organise rapidement autour de lui. Il y a également ces " revues d'actualité ", ces spectacles hétéroclites enfilant des scènes variées le long d'un fil conducteur généralement assez simple et qui traiteront avec légèreté de différents sujets d'actualité. D'importation française, c'est une formule qui sera adaptée au contexte canadien-français et, notamment, au parler populaire. Comme le notent bien les auteurs, l'utilisation fréquente du personnage de l'habitant des campagnes découvrant la ville illustre le rôle que jouent les revues dans l'appropriation d'une certaine modernité urbaine. Le chapitre suivant, consacré à l'étude du riche contenu des revues, le confirme aisément. Les nouvelles technologies, et spécialement l'électricité, les grandes questions sociales ou morales, et notamment la prohibition, ainsi que l'avènement de la société de consommation avec ses grands magasins sont au cœur des spectacles présentés dans le cadre des revues. Dans ce contexte, on ne peut que 
déplorer l'état très fragmentaire des sources. À partir des quelques textes de revues qui nous sont parvenus, les auteurs nous donnent un aperçu de la densité et de la complexité des représentations du monde moderne transmises lors de ces événements courus. Finalement, un dernier chapitre permet à Lacasse, Massé et Poirier de s'attarder plus longuement sur le personnage d'Alexandre Silvio, dont la trajectoire est intimement liée à ces divertissements populaires. Ils s'arrêtent également sur celui qui pourrait être l'exact opposé de Silvio, le «bonimenteur académique " Joseph Dumais. Le premier permet de suivre la montée et le déclin des revues d'actualité ainsi que les stratégies commerciales et publicitaires mises en avant pour en assurer le succès; alors que le second nous permet de mieux comprendre l'attitude souvent méprisante réservée par l'élite culturelle à ces divertissements populaires.

Lorsque Silvio meurt, en 1935, l'âge d'or des revues d'actualité est déjà bel et bien révolu. Le cinéma parlant et d'autres formes de divertissement populaire prennent la relève. L'ouvrage de Lacasse, Massé et Poirier permet néanmoins de redonner sa place dans l'historiographie à cet épisode coloré et riche de l'histoire culturelle de Montréal et de prendre la mesure des différentes avenues empruntées par les Canadiens français pour apprivoiser la modernité urbaine au cours de la première moitié $\mathrm{du} \mathrm{xx}^{\mathrm{e}}$ siècle.

- Harold Bérubé

Université de Sherbrooke

\section{Frédéric Smith. «La France appelle votre secours » : Québec et la France libre, 1940-1945, Montréal, VLB éditeur, 2012, 293 p.}

Frédéric Smith, historien pour la Commission de la capitale nationale à Québec, produit ici un livre intéressant et qui poursuit le travail amorcé depuis plusieurs années par Éric Amyot, Sébastien Vincent, Olivier Courteaux et d'autres, sur les liens des Canadiens français avec le régime de Vichy pendant la Seconde Guerre mondiale. On 